Positive streamers in air of varying density: experiments on the scaling of the excitation density

This content has been downloaded from IOPscience. Please scroll down to see the full text. 2015 J. Phys. D: Appl. Phys. 48055205

(http://iopscience.iop.org/0022-3727/48/5/055205)

View the table of contents for this issue, or go to the journal homepage for more

Download details:

IP Address: 131.155.2.68

This content was downloaded on 30/01/2015 at 17:23

Please note that terms and conditions apply. 


\title{
Positive streamers in air of varying density: experiments on the scaling of the excitation density
}

\author{
D Dubrovin ${ }^{1,2}$, S Nijdam ${ }^{3}$, T T J Clevis ${ }^{3}$, L C J Heijmans ${ }^{3}$, U Ebert ${ }^{3,4}$, Y Yair ${ }^{2,5}$ \\ and C Price ${ }^{1}$
}

${ }^{1}$ Department of Geophysical, Atmospheric and Planetary Sciences, Tel-Aviv University, Tel-Aviv, Israel

2 Department of Natural Sciences, The Open University of Israel, Ra'anana, Israel

${ }^{3}$ Department of Applied Physics, Eindhoven University of Technology, Eindhoven, The Netherlands

${ }^{4}$ Centre for Mathematics and Computer Science (CWI), Amsterdam, The Netherlands

5 School of Sustainability, Interdisciplinary Center Herzliya (IDC), Herzliya, Israel

E-mail: yoav.yair@idc.ac.il

Received 10 October 2014, revised 1 December 2014

Accepted for publication 22 December 2014

Published 19 January 2015

\begin{abstract}
Streamers are rapidly extending ionized finger-like structures that dominate the initial breakdown of large gas volumes in the presence of a sufficiently strong electric field. Their macroscopic parameters are described by simple scaling relations, where the densities of electrons and of excited molecules in the active streamer front scale as the square of the density of the neutral gas. In this work we estimate the absolute density of nitrogen molecules, excited to the $\mathrm{C}^{3} \prod_{\mathrm{u}}$ state that emit photons in the $2 \mathrm{P}-\mathrm{N}_{2}$ band, by radiometrically calibrated short exposure intensified imaging. We test several pressures (100, 200 and 400 mbar) in artificial air at room temperature. Our results provide a first confirmation for the scaling of the density of excited species with the gas density. The method proposed here is particularly suitable to characterize the excitation densities in sprite streamers in the atmosphere.
\end{abstract}

Keywords: streamers, radiance, excitation density, sprites, electron density

(Some figures may appear in colour only in the online journal)

\section{Introduction}

The initial breakdown of large gas volumes is dominated by rapidly extending ionized finger-like transient structures known as streamers, which occur in the presence of a sufficiently strong electric field. They appear on a large scale in the mesosphere, where they are observed in sprites, and on a smaller scale in streamer discharges created in laboratory settings at standard atmospheric density. Streamer discharges have the appearance of a tree like structure, with streamers branching to all sides from a few main channels (recently modelled by Luque and Ebert [1]). The macroscopic parameters of streamers are described by simple scaling relations; the electric field in the streamer scales as the neutral density $N$, the diameter as $1 / N$, and the densities of produced species (such as free electrons, ions, radicals and excited molecules) scale as $N^{2}$ [2-5]. Streamer diameters, velocities and inferred electric fields, measured in the laboratory at close to standard temperature and pressure were shown to scale with gas density to sprite streamers [6-9].

The absolute densities of the various species $n_{x}$ produced by the passage of streamers in pure nitrogen and air were measured with several methods, typically at standard pressure (see e.g. [10-17]). The parameter which is typically estimated in these measurements is the bulk production of active species. The volume of gas treated by a streamer discharge is estimated using the number of streamers and their mean diameter, as derived from images of the entire discharge. The density of excited molecules (the excitation density) produced by a single streamer is then estimated as the ratio between the bulk production and the average volume treated by the discharge, which consists of numerous streamers. Šimek et al [12] estimate the 
density of the $\mathrm{N}_{2}\left(\mathrm{~A}^{3} \Sigma_{\mathrm{u}}^{+}\right)$and $\mathrm{N}_{2}\left(\mathrm{C}^{3} \Pi_{\mathrm{u}}\right)$ excited states, produced by the passage of a streamer discharge in high purity nitrogen (quality not specified) and their decay over time, using the Herman infrared emission (HIR) technique. They estimated the excitation densities a short time after the discharge, finding $\sim 5 \times 10^{14} \mathrm{~cm}^{-3}$ at $\sim 1 \mu$ s after the discharge and $\sim 10^{13} \mathrm{~cm}^{-3}$ less than $0.1 \mu \mathrm{s}$ after the discharge, respectively. Ono et al [15] used the Laser-Induced Fluorescence (LIF) technique to estimate that the density of $\mathrm{N}_{2}\left(\mathrm{~A}^{3} \Sigma_{\mathrm{u}}{ }^{+}\right)$created in the path of a streamer in pure nitrogen is $\sim 6 \times 10^{13} \mathrm{~cm}^{-3}$. That work was followed by Teramoto et al [16], who estimated the absolute density of $\mathrm{N}_{2}\left(\mathrm{~A}^{3} \Sigma_{\mathrm{u}}^{+}\right)$in nitrogen with trace amounts of additives and found that while in pure nitrogen the absolute density is $2-3 \times 10^{13} \mathrm{~cm}^{-3}$, when more than $0.1 \%$ oxygen is added the excitation density grows to $\sim 4 \times 10^{13} \mathrm{~cm}^{-3}$. They also found that the density of the excited molecules increases by a factor of 2 when the applied voltage increases from 18.5 to $21.5 \mathrm{kV}$. Naidis [18] reexamined these experimental results, taking into account the strong radial non-uniformity of plasma parameters in the streamer channel. His estimation of the initial number density of $\mathrm{N}_{2}\left(\mathrm{~A}^{3} \Sigma_{\mathrm{u}}^{+}\right)$on the axis of the streamer was $\sim 4-9 \times 10^{14} \mathrm{~cm}^{-3}$. Pancheshnyi et al [10] measured the excitation densities of several excited states, among them the $\mathrm{N}_{2}\left(\mathrm{C}^{3} \Pi_{\mathrm{u}}\right)$ state, by analyzing the emission spectrum along the path of the discharge. They estimated that the maximum linear density of the $\mathrm{N}_{2}\left(\mathrm{C}^{3} \Pi_{\mathrm{u}}\right)$ excitations was $0.5-1.0 \times 10^{10} \mathrm{~cm}^{-1}$. The radius of a streamer can vary by an order of magnitude when the applied voltage is doubled [19]. Assuming, for the sake of argument, that the streamer diameter is $0.4 \mathrm{~mm}$ (not a minimal streamer) at standard pressure the excitation volume density can be estimated as $4-8 \times 10^{12} \mathrm{~cm}^{-3}$. Additional insight can be gained from the guided streamers (plasma bullets), recently reviewed by Lu et al [20]. Experimental estimations of the electron density in the streamer column of the plasma bullet are $2 \times 10^{13} \mathrm{~cm}^{-3}$ [21] and $10^{11}-10^{13} \mathrm{~cm}^{-3}$ [22] Unlike the streamers discussed in this work, guided streamers follow a known trajectory determined by pre-ionization, which is achieved by a laser beam or a thin column of a plasma jet injected in the discharge gap.

The production of various excited species and the increase of the electron density due to the passage of a streamer were simulated in several papers [3, 23-33], to name but a few, most of which agree that the electron density on the axis of the streamer is on the order of $10^{13}-10^{14} \mathrm{~cm}^{-3}$. In general, the density of the excited species on the streamers axis will depend both on the neutral gas density and on the maximal electric field at the streamer tip. Naidis [23] and later Li et al [34] proposed an approximation for the production of species, expressed as:

$$
n_{x}=\frac{\epsilon_{0}}{e} \int_{0}^{\left|E^{+}\right|} \alpha_{x}(E) \mathrm{d} E
$$

where $n_{x}$ is the density of produced species, $\alpha_{x}$ is the inverse ionization or excitation length and $\left|E^{+}\right|$is the maximal electric field ahead of the streamer front. Using (1) it is easy to see that the densities of many species on the axis of the streamer

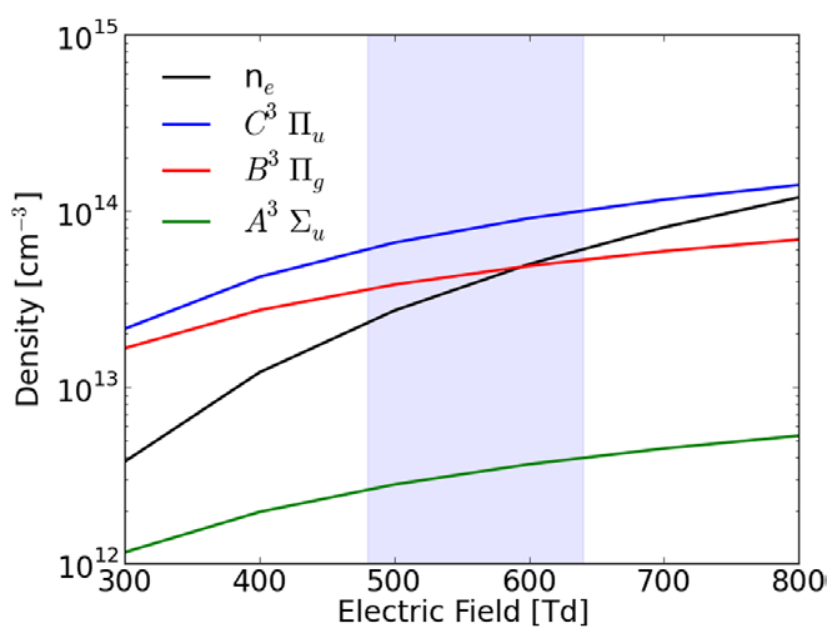

Figure 1. Densities of electrons, $n_{e}$, and excited molecules produced by the passage of a streamer in artificial air $\left(\mathrm{N}_{2}: \mathrm{O}_{2-}\right.$ $80: 20)$. The shaded area indicates the range of the electric field in the streamer front according to Naidis [8] (480-640Td). Densities were calculated with equation (1). Excitation rates were calculated with the BOLSIG+ software [36]. Cross sections were taken from $[37,38]$ and references therein.

are of the same order of magnitude as the density of free electrons, and all depend on the locally enhanced electric field. Naidis [8] reviewed previous work and found that the maximal electric field at the streamer head in laboratory settings at 1 bar is $120-160 \mathrm{kV} \mathrm{cm}^{-1}$, corresponding to 480-640 Td (1 Td $\left.=10^{-17} \mathrm{~V} \cdot \mathrm{cm}^{2}\right)$. The densities of several excited species, calculated with equation (1), are plotted in figure 1 . This plot shows the densities produced by electron impact excitation during the passage of a streamer. The electron density and the $\mathrm{N}_{2}\left(\mathrm{C}^{3} \Pi_{\mathrm{u}}\right)$ density vary by a factor of $4-5$ in this range, with $2-7 \times 10^{13}$ and $0.6-1.0 \times 10^{13} \mathrm{~cm}^{-3}$ respectively. It should be noted, that in our experiments, we do not observe streamers which are wider than $2 \mathrm{~mm}$ bar. With this limitation the electric field fits into a smaller range than proposed by Naidis [8], $120-140 \mathrm{kV} \mathrm{cm}^{-1}(480-560 \mathrm{Td})$, where the densities vary by a factor of two.

As the excited molecules decay to lower states, the densities of low lying excited states increase. The second positive system $\left(2 \mathrm{PN}_{2}\right)$ is the result of the transition $\mathrm{C}^{3} \Pi_{\mathrm{u}} \rightarrow \mathrm{B}^{3} \Pi_{\mathrm{g}}$, occurring on the time scale of $50 \mathrm{~ns}$; the $\mathrm{B}^{3} \Pi_{\mathrm{g}}$ state decays in turn into the $\mathrm{A}^{3} \Sigma_{u}$ state, while emitting in the first positive system $\left(1 \mathrm{PN}_{2}\right)$, on the time scale of $\sim 6 \mu \mathrm{s}$. The $\mathrm{A}^{3} \Sigma_{u}$ state decays on the time scale of $1 \mu \mathrm{s}$. The plot in figure 1 represents the initial excitation densities, not including subsequent transitions. Time scales are taken from Kuo et al [35].

\section{Scaling of excitation densities}

The processes that drive streamer propagation are determined primarily by two-body interactions, the collisions of free electrons with heavy molecules (in weakly ionized plasmas, collisions with ions or other electrons are rare). The length scale of the discharge process in the streamer tip is the mean free path of electrons. Since electrons collide mostly with neutral molecules, the mean free path of 
electrons is inversely proportional to the neutral number density $N$. Therefore all lengths and times determined by electron motion scale like $1 / N$. The electric field in front of the streamer head follows the Townsend scaling relation, $E \sim N$. The Poisson equation leads to the scaling relation of the charge density, $\rho=\nabla \mathbf{E} \sim E / d$, so that the charge density, the density of electrons and ions, as well as the density of the products of electron-molecule collisions, all scale with $N^{2}$. The topic of scaling relations in streamers is discussed in detail by Ebert et al [39]. We do not treat photo-ionization here, as several works demonstrated that this effect can be neglected at ground pressure. Photo-ionization may result in the breaking of scaling when laboratory streamers are compared with sprites $[25,30,40]$.

Scaling of streamer diameters and velocities was demonstrated in experiments in air and in other gas mixtures $[7,27,40,41]$. The density of molecules excited by the passage of a streamer is related to the amount of light emitted in the streamer head. Therefore it is possible to test the scaling of excitation densities, and estimate their absolute value at different pressures using calibrated radiometrical measurements. Pancheshnyi et al [27] measured the integral radiation of a streamer discharge as a function of pressure (in relative units). They found that as pressure increases from 300 to 760 Torr (400-1000 mbar), the emission intensity decreases, indicating that the total production of $\mathrm{N}_{2}\left(\mathrm{C}^{3} \Pi_{\mathrm{u}}\right)$ increases with decreasing pressure. According to Ebert [39]), the excitation and electron density integrated over the streamer head scales like $1 / N$.

Streamers are a transient phenomenon, observed optically as the passage of a bright front, the streamer head, propagating at velocities between $10^{5}$ and $10^{7} \mathrm{~m} \mathrm{~s}^{-1}[9,19,26,41,42]$. As the streamer propagates in the discharge gap, passing in the field of view of the camera, it illuminates several pixels in the image for less than $1 \mathrm{~ns}$ (and only a few microseconds in sprites), typically only a fraction of the camera exposure time. We do not use the more common measure of radiance in Rayleigh units as this quantity requires knowledge of the illumination duration, which is typically shorter than the exposure time in our experiments, as well as in sprite observations. If a source is well resolved spatially, but not well resolved temporally, radiance measurement will depend on the exposure time, as was demonstrated in [26]. Therefore the relevant quantity when discussing the brightness of streamers is the time-integrated radiance (TI-radiance), a measure of the total number of photons emitted from a gas volume into a cone defined by a unit area and a solid angle.

In this work we test the scaling of the density of excited molecules, created by the passage of primary positive streamers in a point plane discharge gap. We measure the radiance and diameters of individual streamers and estimate the excitation density of nitrogen molecules in the $\mathrm{C}^{3} \Pi_{\mathrm{u}}$ state, at several pressures (e.g. 100, 200 and 400 mbar) in artificial air at room temperature. This state emits photons in the second positive system $\left(2 \mathrm{PN}_{2}\right)$. We measure the TI-radiance of streamers imaged by a fast, radiometrically calibrated ICCD camera (Stanford 4QuickE). In this manner we can estimate the excitation density produced by individual streamers, rather than the average production of many streamers. To our knowledge this has never been tested experimentally.

Assuming that emitting molecules radiate isotropically and are distributed uniformly in a cylindrical volume, and the streamer is optically thin, then the maximum TI-radiance of a streamer is the density of photon-emitting molecules integrated along the line of sight through the center of the cylindrical volume:

$$
L_{t} \sim d n^{*} Q / 4 \pi
$$

where $d$ is the optical (observed) diameter of the streamer, $n^{*}$ denotes the density of the excited molecules, $Q$ is the fraction of the excited molecules that undergo radiative relaxation, and the factor $4 \pi$ accounts for isotropic emissions. In the experiments described here the optical emissions are dominated by the $2 \mathrm{PN}_{2}$ band in the near UV, created by the transition $C^{3} \Pi_{u} \rightarrow B^{3} \Pi_{g}[10]$. Some of the excited molecules lose their energy through collisional de-excitation by other molecules. The quenching factor $Q=\left(1+N / N_{q}\right)^{-1}$ is the fraction of excited states that emit photons. Here $N$ is the neutral density and $N_{q}$ is the quenching density. For the $\mathrm{N}_{2}\left(\mathrm{C}^{3} \Pi_{\mathrm{u}}\right)$ state $N_{q}$ is $2.9 \times 10^{17} \mathrm{~cm}^{-3}$, equivalent to $p_{q}=12 \mathrm{mbar}$ at standard temperature. When the neutral density is equal to the quenching density half of the excited molecules emit photons. We used the values listed in Kuo et al [35].

It is possible to measure the TI-radiance and the streamer diameter, and derive the density of excited species as

$$
n^{*} \sim 4 \pi L_{t} / Q d
$$

If $n^{*}$ does not depend strongly on the thickness of the streamers, then $L_{t}$ depends linearly on diameter. According to an analysis by Naidis [8], based in part on measurements by Briels et al [19] and Winands et al [42], the reduced electric field in the streamers' heads does not depend strongly on the diameter of the streamers. In our setup, which follows [19], we do not observe streamers with reduced diameters wider than $2 \mathrm{~mm}$ bar, which makes us conclude that the electric field in the streamer head is fairly constant. It is therefore reasonable to expect a weak dependency of $n^{*}$ on the diameter. In the present analysis we neglect the non-uniform radial distribution of $n^{*}$ in the streamer channel (the implication of a non-uniform radial distribution on streamer emissions is discussed in detail by Nudnova and Starikovskii [43]).

\section{Experimental methods}

A complete description of the experimental setup can be found in $[40,44]$ and will be repeated here briefly. We create positive streamers in a $16 \mathrm{~cm}$ point-plane gap, contained within a large cylindrical stainless steel vacuum vessel, specifically designed to maintain the purity of the enclosed gases. The vessel is filled with artificial air, $\mathrm{N}_{2}: \mathrm{O}_{2}-80 \%: 20 \%$, the pressure is controlled in the range of $100-1000 \mathrm{mbar}$ at room temperature. We used three different pressures: 100, 200 and 400 mbar. A high voltage pulse was generated by a so called C-supply, described fully by Briels et al [44]. It has a 10-90\% 

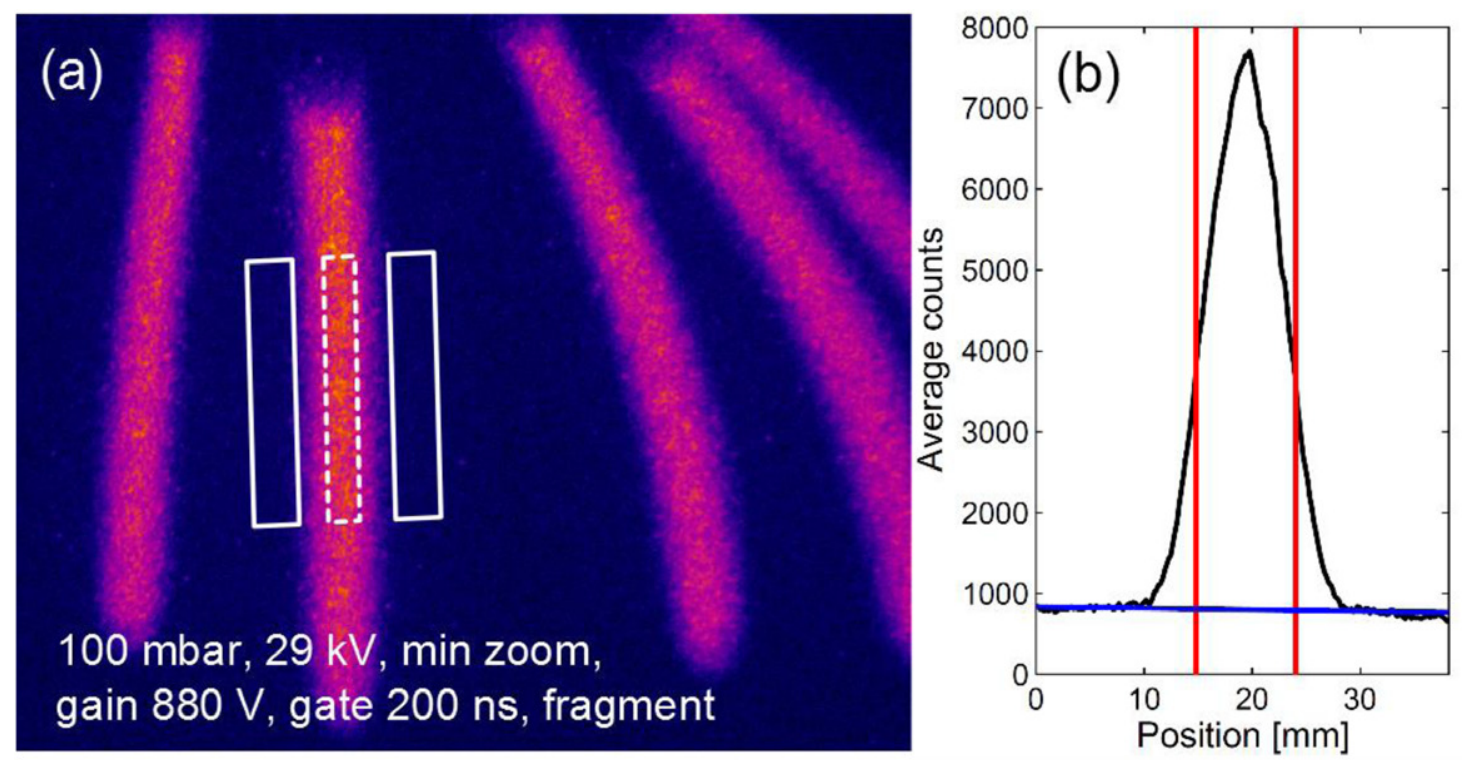

Figure 2. (a) A short exposure image of a streamer in dry air, rendered in false color. Rectangles indicate the regions of interest used to find the mean gray level of the streamer (GL, dashed), and the background $\left(\mathrm{GL}_{0}\right.$, solid). (b) The mean cross section of the streamer in $(a)$. The full width at half maximum (FWHM, confined by two vertical lines) of the cross section determines the width of the region of interest. GL is indicated by the horizontal line.

rise time between 70 and $120 \mathrm{~ns}$ and a fall time of about $10 \mu \mathrm{s}$. The peak of the voltage pulse can be set to values between 10 and $40 \mathrm{kV}$. The higher voltage peaks typically result in the formation of thicker streamers near the needle electrode and in the discharge gap.

A quartz window is installed in the vacuum vessel to allow viewing and recording of the discharge. The vessel and circuit are placed in an external Faraday cage which protects the delicate imaging equipment, with a viewing window made of a fine wire mesh grid to complete the Faraday cage. The fine mesh grid window is equally transparent to all wavelengths in the visible and the near UV range, transmitting $~ 52 \%$ of the light.

The streamers are imaged using a Stanford Computer Optics 4QuickE intensified CCD camera, with nanosecond exposure time, mounted with a UV-Nikkor $105 \mathrm{~mm}$ lens, sensitive to wavelengths in the range $120-850 \mathrm{~nm}$. The imaging setup was calibrated using a tungsten ribbon lamp, as described in the appendix.

The estimation of the brightness of a streamer is illustrated in figure 2: a straight isolated streamer channel section is selected in the image. Several perpendicular cross sections along the chosen streamer are averaged to obtain a single curve, as shown in figure $2(b)$. The streamer diameter is determined as the full width at half maximum (FWHM) of the peak. A rectangular region of interest (ROI) is chosen along the streamer center, with width FWHM (dashed box in figure 2(a)). An average gray level (GL) value is calculated within the ROI. Assuming the GL is distributed as a Gaussian in the radial direction, the average GL determined in this manner is about $80 \%$ of the value determined at the peak. The background gray level $\left(\mathrm{GL}_{0}\right)$ is determined using two ROI parallel and outside of the streamer section with the same dimensions (solid boxes in figure 2(a)). A similar method to
Table 1. Camera zoom settings and related optical lengths. $R$ is the distance between the front of the camera and the needle electrode, DoF is the depth of field, calculated for a circle of confusion of 10 pixels and $m$ is the magnification. The error in magnification is representative of the estimation error of FWHM.

\begin{tabular}{lcll}
\hline Zoom & $R[\mathrm{~mm}]$ & DoF $[\mathrm{cm}]$ & $m$ \\
\hline Max & 620 & 1.6 & $0.28 \pm 1.6 \%$ \\
Mid & 1130 & 8 & $0.12 \pm 0.6 \%$ \\
Min & 2050 & 30 & $0.06 \pm 4 \%$ \\
\hline
\end{tabular}

determine the background signal was used by Yaniv et al [45]. Several hundred streamer sections were analyzed in this manner at each pressure.

The camera was moved on an optical rail to one of three positions, with different distances from the discharge vessel (indicated in table 1 as $R$ ). The position of the camera determines the instantaneous field of view $\Omega_{\mathrm{IFOV}}$, the magnification, and critically, the depth of field. The radiance of an extended source on the other hand, does not depend on the position of the camera. Streamers in our analysis are wider than 10 pixels, and can be considered as extended sources. The depth of field describes how far from the focal plane an object can be placed to appear focused in the image. It depends on the resolution of the camera and the distance from the object. The estimated values depths of field are listed in table 1 . When the depth of field is narrower than the volume of the vessel, many streamers are out of focus. As a result their image on the CCD appears wider, and the measured diameter is overestimated. It is usually impossible to distinguish between streamers that are in focus and those that are not. On the other hand, if the camera is positioned far from the discharge, increasing the depth of field, the imaged streamers are too narrow to be resolved. To overcome these 


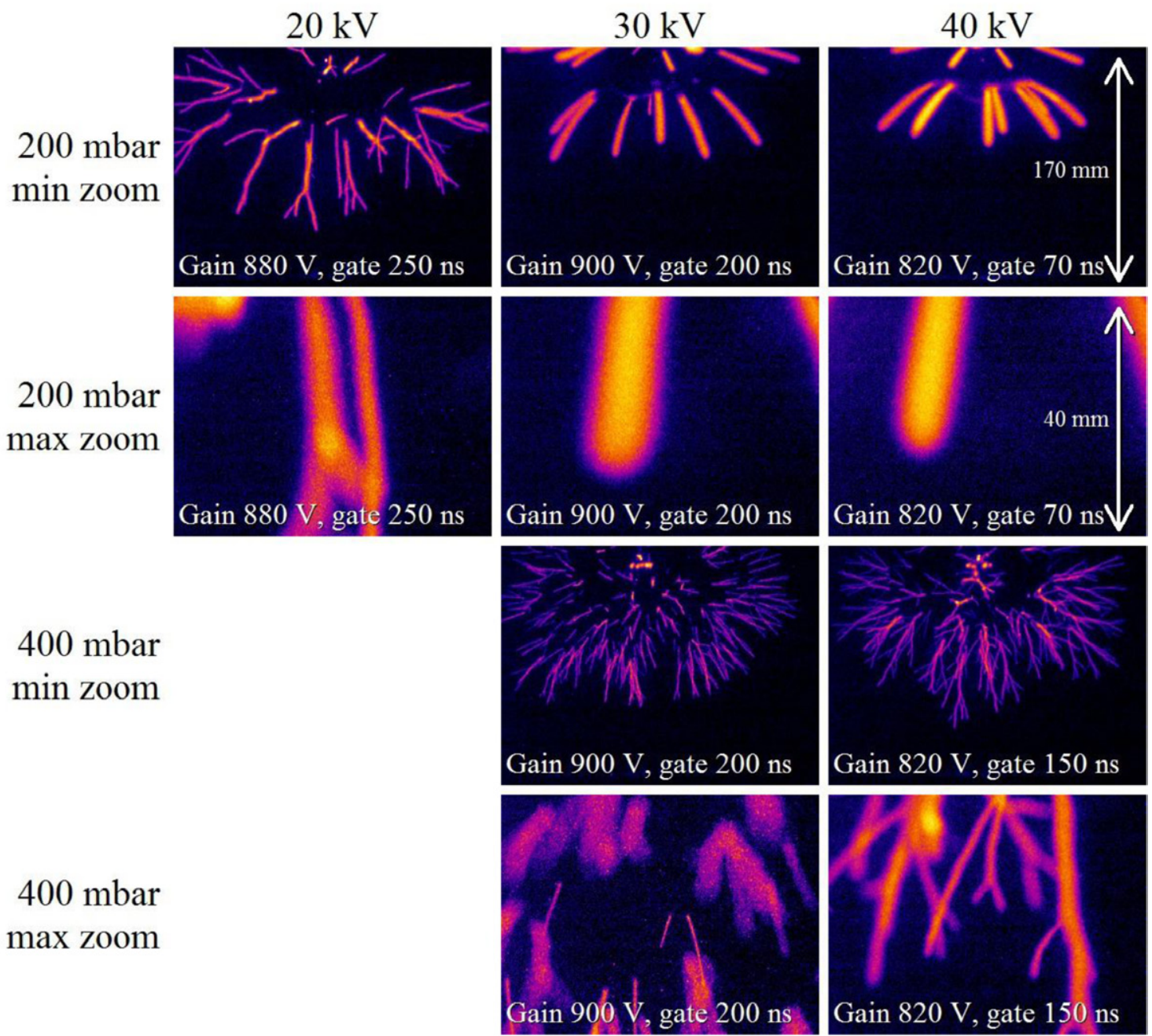

Figure 3. Sample images of streamer discharges at 200 and 400 mbar. The columns correspond to applied voltages as indicated above. The rows correspond to pressures and zoom settings. The gain voltage and exposure time are indicated in each image. At the min zoom setting the entire discharge volume is observed, with the needle at the top of the image. Streamers emanate either from the tip of the needle or from sharp edges above it. The latter do not typically propagate in the direction of the plate, and are not included in the analysis below. The plate is located at the bottom of the image (a weak reflection of the discharge can be observed there). At the max zoom setting neither electrode appears in the image. The depth of field is narrow, with an obvious example in $400 \mathrm{mbar}, 30 \mathrm{kV}$.

limitations we used several camera positions, indicated by min, mid and max zoom in table 1 and figure 3.

Sample images at various voltage settings and camera positions are shown in figure 3. At the minimum zoom setting the entire discharge volume from needle to plate electrode is observed. The resolution of these images is reasonable for the 100 and 200 mbar settings, but at 400 mbar the streamers are narrower than 10 pixels, and their diameters cannot be determined reliably. At maximum zoom only a small volume midway between the electrodes is imaged. With this setting we encounter a different problem - the depth of field is extremely narrow. For relatively wide streamers at low pressures this probably leads to some overestimation of the measured diameters. At 400 mbar streamers are narrow, which may lead to very significant widening of the imaged streamer, up to an order of magnitude. These extremely overestimated streamers are easily distinguished and are omitted from the analysis. Streamers at pressures higher than 400 mbar are even narrower, and we were unable to resolve them.

\section{Results}

In figure 4 we plot the time-integrated radiance $L_{t}$ as function of the reduced diameter $p d$ for each pressure. We find a positive correlation between $L_{t}$ and $p d$ in the three datasets, with the linear Pearson coefficient between 0.6 and 0.7 (see table 2). In figure 4(a) measurements at all three pressures overlap, implying that the radiance of streamers at different pressures but similar conditions, follows a similar trend.

To test whether the time integrated radiance depends linearly on diameter, as expected, we perform a linear fit to the $\log -\log$ plot of $L_{t}$ versus $p d$ (figures $4(b)-(d)$ ). The slope $\alpha$ is the exponent in the relation $L_{t} \sim(p d)^{\alpha}$, and we expect $\alpha=1$. The fitting results are listed in table 2 . We find that $\alpha<1$, seemingly indicating that less light (photons) is emitted from thicker streamers. However this less-than-linear dependence can also be explained by the setup limitations: when the camera is close to the discharge, its depth of field is very narrow. Streamers which are not in the focal plane may appear wider, leading 

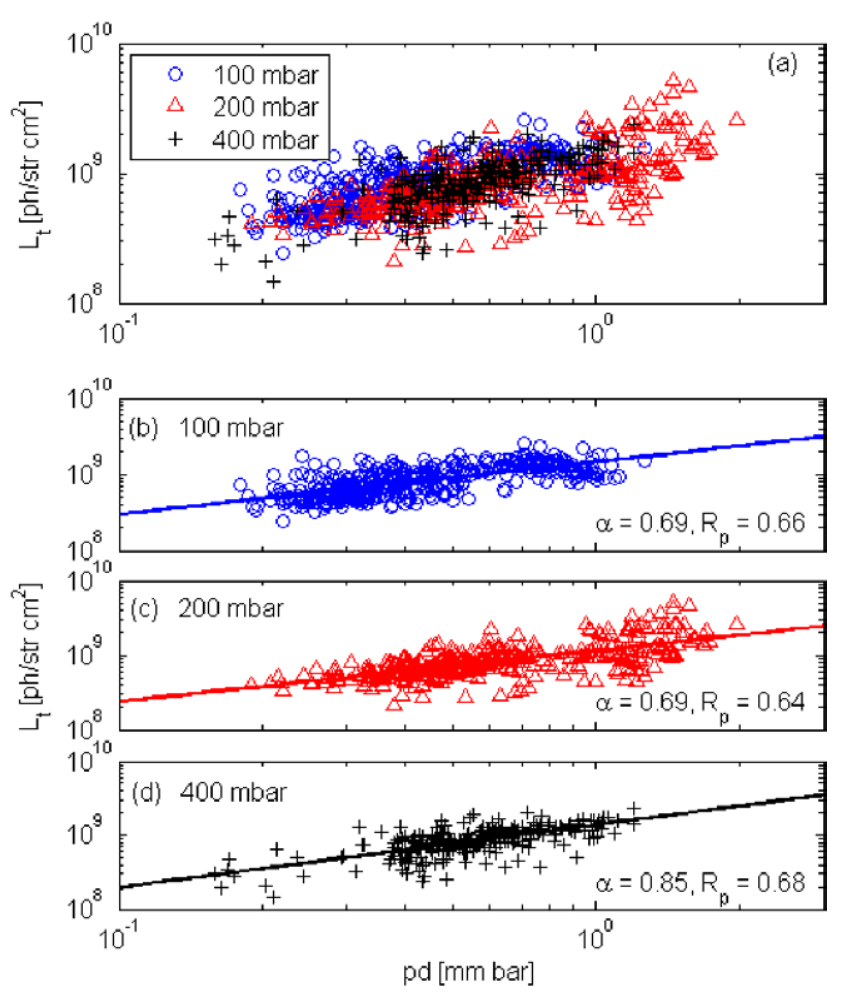

Figure 4. (a) TI-radiance is plotted versus the reduced diameter, showing the measurement results at all three pressures. $(b)-(d)$ Linear fitting to the $\log -\log$ plots of $L_{t}$ versus $p d$. The exponent $\alpha$ in $L_{t} \propto(p d)^{\alpha}$, and the linear Pearson correlation $R_{p}$ for each pressure is indicated in the plots.

to an overestimation of their diameter. On the other hand as long as the image of an out of focus streamer is not extremely blurred, the radiance measurement on its central axis should not be significantly affected. This would cause some of the data points to appear below the expected linear trend line.

The density of excited species $n^{*}$ is estimated using the TI-radiance measurement and equation (3). Under the assumption that most of the emitted photons are in the $2 \mathrm{PN}_{2}$ band, $n^{*}$ is an estimation of the density of the $\mathrm{N}_{2}\left(\mathrm{C}^{3} \Pi_{\mathrm{u}}\right)$ excited state, in which case the quenching density $N_{q}$ is $2.93 \times 10^{17} \mathrm{~cm}^{-3}$. In figure 5 we plot $n^{*}$ as a function of the reduced diameter $p d$. Our first observation in this plot is that the correlation of $n^{*}$ with diameter is weak. It appears that in the streamers in this experiment the density of excited species does not change significantly with streamer width. Our estimation of the mean $n^{*}$ is $(2.4 \pm 0.9) \times 10^{11} \mathrm{~cm}^{-3}$ at $100 \mathrm{mbar},(6.3 \pm 2.4) \times 10^{11} \mathrm{~cm}^{-3}$ at $200 \mathrm{mbar}$, and $(27 \pm 9) \times 10^{11} \mathrm{~cm}^{-3}$ at $400 \mathrm{mbar}$, where the errors are the standard deviation of the average value, typically near $40 \%$. It should be noted that $n^{*}=4 \pi L_{t} / Q d$ and $p d$ are not independently measured variables, while $L_{t}$ and $p d$ are.

In figure 5 it is apparent that when pressure (i.e. neutral density) is higher, the density of excited molecules $n^{*}$ is larger. The density of excited species and electrons in the streamer head should scale as the square of the neutral density, $N^{2}$. Next we estimate the reduced density of excited species, $n^{*} \times\left(p / p_{0}\right)^{-2}$, where $p$ is the pressure and $p_{0}=1000 \mathrm{mbar}$ is a reference pressure. With this scaling the reduced density should be independent of pressure. We plot the averaged reduced density
Table 2. Correlation and fitting parameters as determined in figure 4 . The number of data points is indicated.

\begin{tabular}{llll}
\hline$P$ [mbar] & 100 & 200 & 400 \\
\hline$R_{p}$ & 0.66 & 0.64 & 0.68 \\
$\alpha, \log -\log$ exponent & $0.69 \pm 0.07$ & $0.69 \pm 0.07$ & $0.85 \pm 0.11$ \\
$\begin{array}{l}\text { Number of data } \\
\text { points }\end{array}$ & 415 & 441 & 387 \\
\hline
\end{tabular}

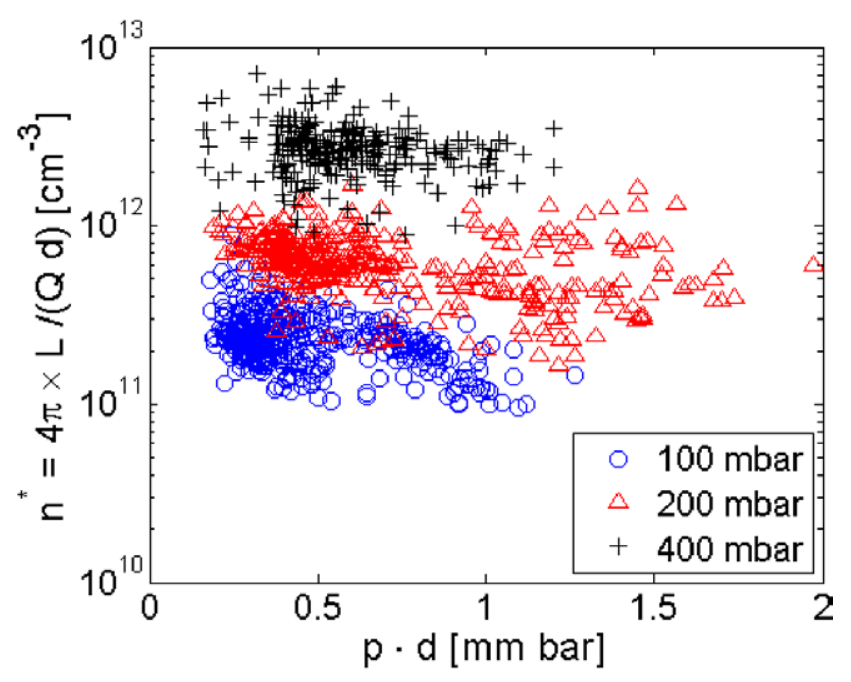

Figure 5. The density of excited species, $n^{*} \sim 4 \pi \cdot L / Q d$, as a function of reduced diameter.

versus pressure in figure 6, where the error bars represent one standard deviation of the averaged values. It appears that within statistical uncertainty the average reduced density is independent of pressure. The measurement at $100 \mathrm{mbar}$ is higher by a factor 1.5 , though it agrees with higher pressure measurements within the standard deviation margin. The reduced density at 200 and 400 mbar is $(1.6 \pm 0.6) \times 10^{13} \mathrm{~cm}^{-3}$ and at $100 \mathrm{mbar}$ it is $(2.4 \pm 0.9) \times 10^{13} \mathrm{~cm}^{-3}$. The average of the reduced density of excited molecules $\left(\mathrm{N}_{2}\left(\mathrm{C}^{3} \Pi_{\mathrm{u}}\right)\right)$ over the three pressures is estimated as $(2 \pm 1) \times 10^{13} \mathrm{~cm}^{-3}$. This is the expected excitation density at 1 bar.

In the introduction we described previous numerical and experimental estimations of the absolute density of electrons, $\mathrm{N}_{2}\left(\mathrm{C}^{3} \Pi_{\mathrm{u}}\right), \mathrm{N}_{2}\left(\mathrm{~A}^{3} \Sigma^{+}{ }_{\mathrm{g}}\right)$ and other species. Model estimations at $1 \mathrm{bar}$ are $10^{13}-10^{14} \mathrm{~cm}^{-3}$. The experimental estimations at 1 bar range from $4 \times 10^{13} \mathrm{~cm}^{-3}$ [16] and up to $9 \times 10^{14} \mathrm{~cm}^{-3}$ [18]. Our estimation of the absolute reduced density is well within the range of previously reported values.

\section{Discussion}

Streamers can be regarded as self-organizing structures, which can be characterized by a few macroscopic parameters. Previous theoretical and experimental work has shown that when parameters such as the neutral density, the velocity, the diameter and the applied voltage are properly scaled, the physical process is essentially the same. This discussion leads to a search of other key characteristics that can be quantified. Numerical models consistently indicate that at standard 


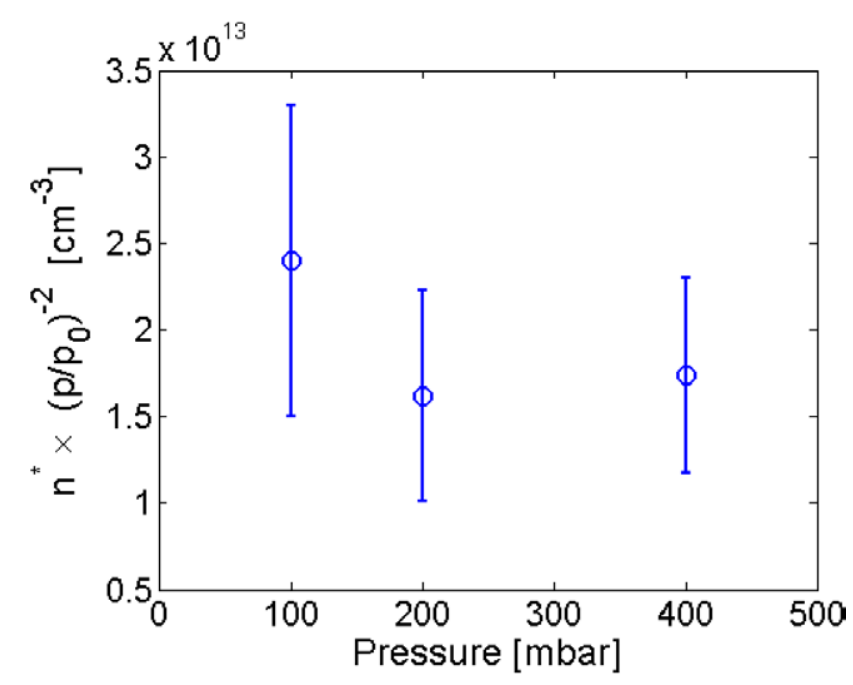

Figure 6. The reduced density of excited species, $n^{*} \times\left(p / p_{0}\right)^{-2}$ as function of pressure. The error bars represent one standard deviation.

pressure, the densities of electrons, and of other products of the passage of the streamer are on the order of $10^{13}-10^{14} \mathrm{~cm}^{-3}$. These findings where confirmed by experimental work. However a rigorous examination of the scaling of the electron density with varying neutral density (gas pressure) has not been done until now. From theoretical considerations the density of electrons produced by the passing streamer head should be proportional to the square of the neutral density $N^{2}$.

We estimate the density of the $\mathrm{N}_{2}\left(\mathrm{C}^{3} \Pi_{\mathrm{u}}\right)$ excited state at three different pressures (100, 200 and $400 \mathrm{mbar})$, and find it is consistent with $N^{2}$ scaling. Following this scaling, our estimation of the excitation density at 1 bar is $(2 \pm 1) \times 10^{13} \mathrm{~cm}^{-3}$. This value is consistent with theoretical and experimental findings at standard pressure. We estimated the time integrated radiance of the streamers as a function of their reduced diameters, $p d$. We expected a linear trend, following equation (2). Our results suggest that the time integrated radiance is proportional to $(p d)^{\alpha}$, with $\alpha=0.69 \pm 0.07$ at pressures 100 and 200 mbar, and $\alpha=0.85 \pm 0.11$ at 400 mbar. Moreover, when the time integrated radiance is plotted against the reduced diameter $p d$, the data points of all three pressures coincide (figure $4(a)$ ). In other words, the trend at different pressures and similar conditions (similar diameters) is similar, a clear signature of scaling relations. We recall here that while the absolute radiance is measured with an error of $30 \%$, the relative radiance is accurate to within 5\% (see appendix A for details). Therefore the statistical distribution observed in the data points is most likely due to differences in the observed streamers, and to some extent, the overestimated diameters of out-of-focus streamers.

It is puzzling that the dependence on $p d$ is not linear (i.e. $\alpha<1)$. We suggest that this is an artifact of the measurement, explained by the overestimation of some streamer diameters when they are out of focus. Out-of-focus streamers can be distinguished from focused streamers only if they are significantly wider than focused streamers in the image. This happens for at least some of the streamers imaged at 400 mbar. This puzzle can be better answered experimentally if the focus artifact is resolved, for example by observing streamers in sprites $[9,46$, 47], by using stereo-photographic imaging [48] to determine the $3 \mathrm{D}$ position of streamers and thereby their distance to the focal plane, or with guided streamers, where the trajectory of the streamer is determined by the setup [20,49].

Alternative explanations would be related to the physics of the discharge. Streamers are optically thin, and the quenching factor $\mathrm{Q}$ is not related to diameter; therefore the measurements may suggest that $n^{*}$ is lower for thicker streamers. Is it possible that the production of excited nitrogen molecules is lower in thicker streamers? According to Naidis [23] and Li et al [34] the densities depend on the electric field, with higher fields leading to higher production of excited molecules (equation (1)). However, the densities predicted by this model in the range of fields relevant to streamer heads [8] are not very sensitive to the electric field, changing by a factor 4 at most, as was shown in figure 1. Our results suggest that the variability of the excitation density in streamer heads may be on a similar scale. The variability of the excitation density may depend on the stochastic nature of the discharge and on local conditions, and should be explored further.

\section{Conclusions}

We presented the results of an experimental investigation of the scaling of the time-integrated radiance of streamers, and the densities of the excited molecules responsible for these emissions. We studied streamer discharges in artificial air, in the pressure range of 100-400mbar. Our results suggest that the time-integrated radiance, defined as the number of photons per unit area per solid angle emitted from a streamer head, depends linearly on the diameter of the streamer. Based on this finding we estimate the density of excited species in the streamer head. We find that this density depends very weakly on the streamer diameter, where wider streamers may have a slightly lower excitation density. This downward trend may be explained by the fact that at least part of the imaged streamers are not in focus.

Interestingly, we find that the radiance and the density of excited molecules exhibit similar trends at different pressures when examined under similar conditions, i.e. when plotted as a function of the reduced diameter $p d$. This is a clear indication of a scaling relation. As we showed, the average density of the molecules excited by the passage of a streamer depends only weakly on the streamer diameter. Therefore we estimated the mean densities of $\mathrm{N}_{2}\left(\mathrm{C}^{3} \Pi_{\mathrm{u}}\right)$ at the available pressures, and the mean reduced density $n^{*} \times\left(p / p_{0}\right)^{-2}=(2 \pm 1) \times 10^{13} \mathrm{~cm}^{-3}$, where $p_{0}=1$ bar. We show that within statistical uncertainty, this excitation density scales as the square of the neutral density.

\section{Acknowledgments}

This work was supported by the Israeli Science Foundation grant $117 / 09$ and by the Ilan Ramon scholarship from the Israeli Ministry of Science. We would like to thank Alejandro Luque for his help with the theoretical calculation of electron and excitation densities in streamers. 


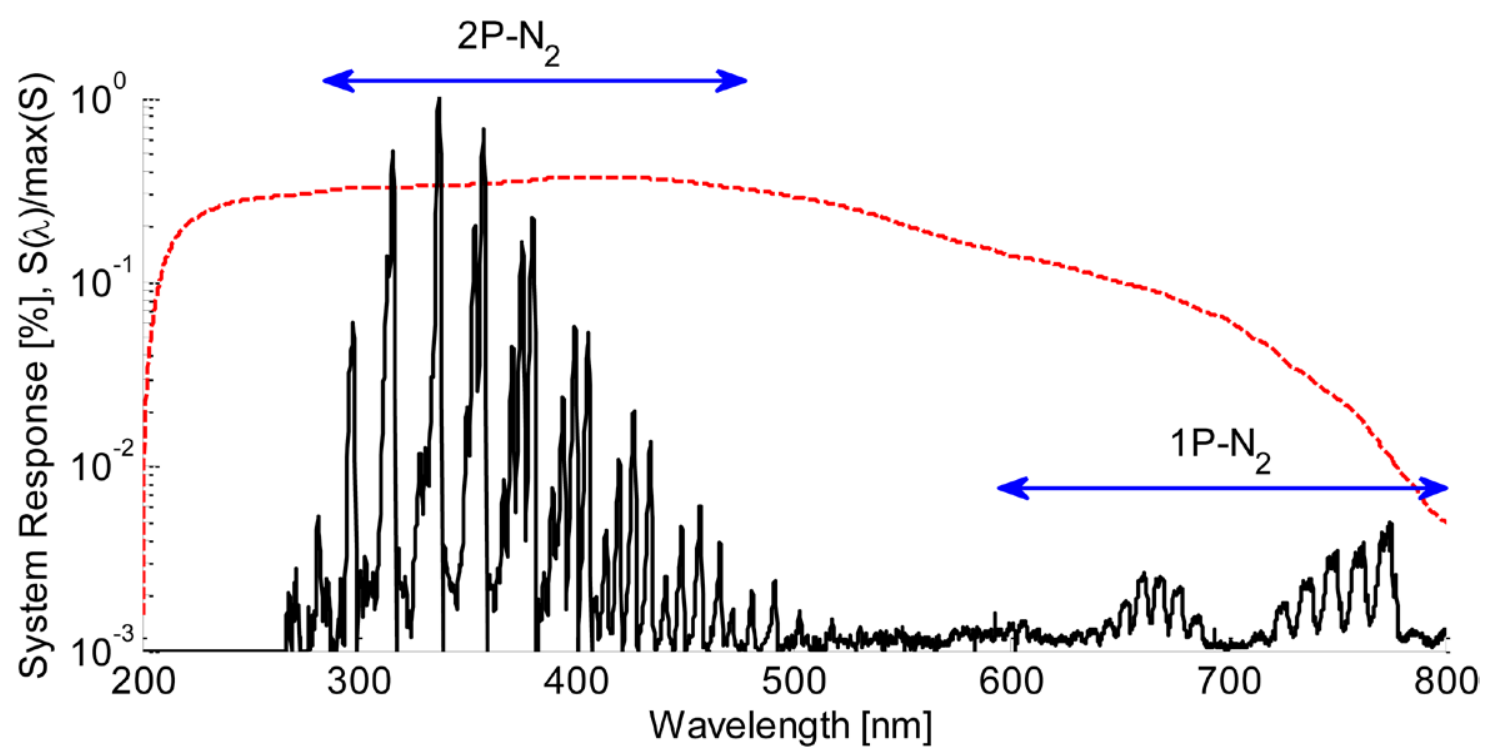

Figure A.1. The spectrum of a glow discharge measured at $25 \mathrm{mbar}$ (solid black), and the combined window transmittance and response functions, $R(\lambda) \cdot W(\lambda)$ (dashed red), shown on a logarithmical scale. The spectrum is normalized so that its maximal value is 1 . The two dominant nitrogen bands, $2 \mathrm{PN}_{2}$ and $1 \mathrm{PN}_{2}$ are indicated. The $1 \mathrm{PN}_{2}$ band is two orders of magnitude weaker than the $2 \mathrm{PN}_{2}$ band, due to collisional quenching.

\section{Appendix A. Description of camera calibration}

The streamers in our setup are imaged using a Stanford Computer Optics 4QuickE intensified CCD camera, with nanosecond exposure time, mounted with a UV-Nikkor $105 \mathrm{~mm}$ lens, sensitive to wavelengths in the range $120-850 \mathrm{~nm}$. The imaging setup was calibrated using a tungsten ribbon lamp. After initial calibration, the radiance of streamers is retrieved from the grey level measured in the ICCD camera. For that purpose the transmittance functions of the quartz and fine wire mesh windows are taken into account. The calibration procedure is described below.

The spectral photon radiance of the tungsten ribbon lamp, $P(\lambda)$, (in photons per solid angle per unit area per second per unit wavelength) is supplied with the lamp. The signal is affected by the response function of the image intensifier in the camera, which depends on the wavelength. The gray level (GL) of an illuminated pixel is therefore

$\mathrm{GL}-\mathrm{GL}_{0}=K \cdot f\left(V_{\mathrm{gain}}\right) \cdot \Omega_{\mathrm{IFOV}} \cdot A_{\mathrm{opt}} \cdot \Delta t \cdot \int P(\lambda) R(\lambda) \mathrm{d} \lambda$,

where $\mathrm{GL}_{0}$ is the background gray level, $K$ is the calibration constant, $f\left(V_{\text {gain }}\right)$ represents the dependency on the intensifier gain voltage, which is controlled externally by the user, $\Delta t$ is the exposure time, $\Omega_{\mathrm{IFOV}}$ is the instantaneous field of view of a pixel and $A_{\text {opt }}$ is the effective collecting area of the lens. The spectral response of the camera is given by $R(\lambda)$, the product of the quantum efficiency of the image intensifier and the transmittance of the UV-Nikkor lens. The quantum efficiency curve is supplied by the manufacturer with an estimated accuracy of $25 \%$, and the transmission of the lens was measured with an accuracy of $20 \%$. The output of this step is the calibration constant $K$. Its absolute value has an error of $\sim 30 \%$. The gain voltage dependence was estimated separately as $f\left(V_{\text {gain }}\right)=$ $\exp \left(a V_{\text {gain }}+b\right)$, where $a=1.4 \cdot 10^{-2} \mathrm{~V}^{-1}$ and $b=-6.6$, with $5 \%$ accuracy. The relative radiance measurement does not depend on the absolute value of $K$, and its accuracy is determined by the accuracy of the gain function $f\left(V_{\text {gain }}\right)$ and the throughput $A_{\text {opt }} \cdot \Omega_{\mathrm{IFOV}}$. This reflects on the accuracy of the correlations we find in our results.

In the 4QuickE ICCD camera the image is formed on a photocathode which intensifies the signal, but also increases the noise. As a result, images less than 10 pixels wide are not well resolved. Each of the $1360 \times 1024$ CCD pixels is represented by an effective pixel of width $d_{\mathrm{px}}=10.575 \mu \mathrm{m}$ on the photo-cathode. The IFOV angle is defined as a $\Omega_{\mathrm{IFOV}}=$ $\left(d_{\mathrm{px}} / u\right)^{2}$, where $u$ is the distance between the image on the photocathode and the effective lens that substitutes the UV-Nikkor lens, such that the lens equation is satisfied.

The strength of the signal produced by the imaged streamers depends on the response function of the camera $R(\lambda)$, the transmittance of the viewing window $W(\lambda)$, and on the normalized spectral distribution of the incoming light $S(\lambda)$. Westimated $S(\lambda)$ by measuring the spectrum of a streamer and glow discharge at low pressures (50-200 mbar, figure A.1). The spectrum was recorded using three small Ocean Optics spectrometers together spanning the wavelength range of $230-940 \mathrm{~nm}$, at several pressures and voltages, similarly to Dubrovin et al [50]. This spectrum is similar to measured and calculated sprite and streamer spectra (see e.g. $[10,51])$. Streamers propagating in air radiate predominantly in the blue and the near UV second positive system $\left(2 \mathrm{PN}_{2}\right)$, in the wavelength range of 300 $400 \mathrm{~nm}$. The red first positive system $\left(1 \mathrm{PN}_{2}\right)$, dominant in ground sprite observations, is quenched at pressures above 1 mbar [35, 52].

The time-integrated radiance $L_{t}=L \cdot \Delta t$ is determined by: 


$$
L_{t}=\frac{\mathrm{GL}-\mathrm{GL}_{0}}{K \cdot f\left(V_{\text {gain }}\right) \cdot \Omega_{\mathrm{IFOV}} \cdot A_{\mathrm{opt}}} \cdot \frac{\int S(\lambda) \mathrm{d} \lambda}{\int S(\lambda) R(\lambda) W(\lambda) \mathrm{d} \lambda},
$$

where $S(\lambda)$ is the spectral distribution of the incoming light (black curve in figure A.1) and $K$ and $f\left(V_{\text {gain }}\right)$ are the calibration constant and gain function determined by the calibration procedure described above. The integration boundaries are determined by the limits of the response function $R(\lambda)$. The $2 \mathrm{PN}_{2}$ band is well within the sensitivity range of the camera. The uncertainty in integration boundaries leads to an error of $\sim 2 \%$. The TI-radiance in this expression is measured in photons per solid angle per unit area $\left(\mathrm{ph} \mathrm{str}^{-1} \mathrm{~cm}^{-2}\right)$. The spectrum of the streamers and the combined transmittance and response curve $R(\lambda) \cdot W(\lambda)$ are plotted in figure A.1.

\section{References}

[1] Luque A and Ebert U 2014 Growing discharge trees with self-consistent charge transport: the collective dynamics of streamers New J. Phys. 16013039

[2] Pasko V P, Inan U S and Bell T F 1998 Spatial structure of sprites Geophys. Res. Lett. 25 2123-6

[3] Liu N and Pasko V P 2004 Effects of photoionization on propagation and branching of positive and negative streamers in sprites J. Geophys. Res. 109 A 04301

[4] Ebert U et al 2006 The multiscale nature of streamers Plasma Sources Sci. Technol. 15 S118-29

[5] Pasko V P 2007 Red sprite discharges in the atmosphere at high altitude: the molecular physics and the similarity with laboratory discharges Plasma Sources Sci. Technol. 16 S13

[6] Ebert U and Sentman D D 2008 Streamers, sprites, leaders, lightning: from micro-to macroscales J. Phys. D Appl. Phys. 41230301

[7] Briels T M P, van Veldhuizen E M and Ebert U 2008 Positive streamers in air and nitrogen of varying density: experiments on similarity laws J. Phys. D Appl. Phys. $\mathbf{4 1} 234008$

[8] Naidis G V 2009 Positive and negative streamers in air: velocity-diameter relation Phys. Rev. E Stat. Nonlinear Soft Matter Phys. 7957401

[9] Kanmae T, Stenbaek-Nielsen H C, McHarg M G and Haaland R K 2012 Diameter-speed relation of sprite streamers J. Phys. D Appl. Phys. 45275203

[10] Pancheshnyi S V, Sobakin S V, Starikovskaya S M and Starikovskii AY 2000 Discharge dynamics and the production of active particles in a cathode-directed streamer Plasma Phys. Rep. 26 1054-65

[11] Niemi K, der Gathen V S and Döbele H F 2005 Absolute atomic oxygen density measurements by two-photon absorption laser-induced fluorescence spectroscopy in an RF-excited atmospheric pressure plasma jet Plasma Sources Sci. Technol. 14 375-86

[12] Šimek M, Člupek M, Babický V and Šunka P 2006 Production of reactive species by atmospheric pressure streamers in $\mathrm{N}_{2}-\mathrm{O}_{2}$ mixtures Pure Appl. Chem. 78 1213-25

[13] Eichwald $\mathrm{O}$ et al 2008 Experimental analysis and modelling of positive streamer in air: towards an estimation of $\mathrm{O}$ and $\mathrm{N}$ radical production J. Phys. D Appl. Phys. 41234002

[14] Ono R, Takezawa K and Oda T 2009 Two-photon absorption laser-induced fluorescence of atomic oxygen in the afterglow of pulsed positive corona discharge J. Appl. Phys. 10643302
[15] Ono R, Tobaru C, Teramoto Y and Oda T 2009 Laser-induced fluorescence of $\mathrm{N}_{2}\left(A^{3} \Sigma_{u}^{+}\right)$metastable in $\mathrm{N}_{2}$ pulsed positive corona discharge Plasma Sources Sci. Technol. 1825006

[16] Teramoto Y, Ono R and Oda T 2009 Measurement of $\mathrm{N}_{2}$ $\left.\left(A^{3} \Sigma_{u}^{+}\right)\right\}$metastable in $\mathrm{N}_{2}$ pulsed positive corona discharge with trace amounts of additives J. Phys. D Appl. Phys. 42 235205

[17] Stancu G D, Kaddouri F, Lacoste D A and Laux C O 2010 Atmospheric pressure plasma diagnostics by OES, CRDS and TALIF J. Phys. D Appl. Phys. 43124002

[18] Naidis G V 2010 Evaluation of N_2 $\left(A^{3} \Sigma_{u}^{+}\right)$density in nitrogen streamer discharges Plasma Sources Sci. Technol. 1955010

[19] Briels T M P, Kos J, Winands G J J, van Veldhuizen E M and Ebert U 2008 Positive and negative streamers in ambient air: measuring diameter, velocity and dissipated energy J. Phys. D Appl. Phys. 41234004

[20] Lu X, Naidis G V, Laroussi M and Ostrikov K 2014 Guided ionization waves: theory and experiments Phys. Rep. $540123-66$

[21] Shashurin A, Shneider M N and Keidar M 2012 Measurements of streamer head potential and conductivity of streamer column in cold nonequilibrium atmospheric plasmas Plasma Sources Sci. Technol. 21034006

[22] Begum A, Laroussi M and Pervez M R 2013 Atmospheric pressure He-air plasma jet: breakdown process and propagation phenomenon AIP Adv. 3062117

[23] Naidis G V 1997 Modelling of plasma chemical processes in pulsed corona discharges J. Phys. D Appl. Phys. 301214

[24] Kulikovsky A A 1997 Production of chemically active species in the air by a single positive streamer in a nonuniform field Plasma Sci. IEEE Trans. 25 439-46

[25] Liu N and Pasko V P 2006 Effects of photoionization on similarity properties of streamers at various pressures in air J. Phys. D Appl. Phys. 39 327-34

[26] Liu N Y, Pasko V P, Adams K, Stenbaek-Nielsen H C and McHarg M G 2009 Comparison of acceleration, expansion, and brightness of sprite streamers obtained from modeling and high-speed video observations J. Geophys. Res. 114 A00E03

[27] Pancheshnyi S, Nudnova M and Starikovskii A 2005 Development of a cathode-directed streamer discharge in air at different pressures: experiment and comparison with direct numerical simulation Phys. Rev. E. 7116407

[28] Pancheshnyi S 2005 Role of electronegative gas admixtures in streamer start, propagation and branching phenomena Plasma Sources Sci. Technol. 14 645-53

[29] Pancheshnyi S, Ségur P, Capeillère J and Bourdon A 2008 Numerical simulation of filamentary discharges with parallel adaptive mesh refinement J. Comput. Phys. 227 6574-90

[30] Wormeester G, Pancheshnyi S, Luque A, Nijdam S and Ebert U 2010 Probing photo-ionization: simulations of positive streamers in varying $\mathrm{N}_{2}: \mathrm{O}_{2}$ mixtures $J$. Phys. $D$ Appl. Phys. 43505201

[31] Komuro A, Ono R and Oda T 2012 Numerical simulation for production of $\mathrm{O}$ and $\mathrm{N}$ radicals in an atmospheric-pressure streamer discharge J. Phys. D Appl. Phys. 45265201

[32] Li C, Ebert U and Hundsdorfer W 2012 Spatially hybrid computations for streamer discharges: II. Fully 3D simulations J. Comput. Phys. 231 1020-50

[33] Luque A, Ratushnaya V and Ebert U 2008 Positive and negative streamers in ambient air: modeling evolution and velocities J. Phys. D Appl. Phys. 41234005

[34] Li C, Brok W J M, Ebert U and der Mullen J 2007 Deviations from the local field approximation in negative streamer heads J. Appl. Phys. 101123305 
[35] Kuo C-L et al 2007 Modeling elves observed by FORMOSAT-2 satellite J. Geophys. Res. Space Phys. 112 A11312

[36] Hagelaar G J M and Pitchford L C 2005 Solving the Boltzmann equation to obtain electron transport coefficients and rate coefficients for fluid models Plasma Sources Sci. Technol. 14 722-33

[37] Phelps A V 2014 BOLSIG+ solver (www.lxcat.net)

[38] Cartwright D C, Chutjian A, Trajmar S and Williams W 1977 Electron impact excitation of the electronic states of N2. I. Differential cross sections at incident energies from 10 to 50 eV Phys. Rev. A 161013

[39] Ebert U, Nijdam S, Chao L, Luque A, Briels T-M-P and van-Veldhuizen E-M 2010 Review of recent results on streamer discharges and discussion of their relevance for sprites and lightning J. Geophys. Res. 115 A00E43

[40] Nijdam S, van de Wetering F M J H, Blanc R, van Veldhuizen E M and Ebert U 2010 Probing photoionization: experiments on positive streamers in pure gases and mixtures J. Phys. D Appl. Phys. 43145204

[41] Gerken E A, Inan U S and Barrington-Leigh C P 2000 Telescopic imaging of sprites Geophys. Res. Lett. 272637

[42] Winands G J J, Liu Z, Pemen A J M, Van Heesch E J M and Yan K 2008 Analysis of streamer properties in air as function of pulse and reactor parameters by $\{$ ICCD $\}$ photography J. Phys. D Appl. Phys. 41234001

[43] Nudnova M M and Starikovskii A Y 2008 Streamer head structure: role of ionization and photoionization J. Phys. D Appl. Phys. $\mathbf{4 1} 234003$
[44] Briels T M P, Kos J, van Veldhuizen E M and Ebert U 2006 Circuit dependence of the diameter of pulsed positive streamers in air J. Phys. D Appl. Phys. 395201

[45] Yaniv R et al 2014 Ground-based observations of the relations between lightning charge-moment-change and the physical and optical properties of column sprites J. Atmos. SolarTerre. Phys. 107 60-7

[46] Stenbaek-Nielsen H C, McHarg M G, Kanmae T and Sentman D D 2007 Observed emission rates in sprite streamer heads Geophys. Res. Lett. 34 L11105

[47] Stenbaek-Nielsen H C, Kanmae T, McHarg M G and Haaland R 2013 High-speed observations of sprite streamers Surv. Geophys. 34 769-95

[48] Nijdam S, Moerman J S, Briels T M P, van Veldhuizen E M and Ebert U 2008 Stereo-photography of streamers in air Appl. Phys. Lett. 92101502

[49] Nijdam S, Takahashi E, Teunissen J and Ebert U 2014 Streamer discharges can move perpendicularly to the electric field New J. Phys. 16103038

[50] Dubrovin D, Nijdam S, Van Veldhuizen E M, Ebert U, Yair Y and Price C 2010 Sprite discharges on Venus and Jupiterlike planets: a laboratory investigation J. Geophys. Res. 115 A00E34

[51] Kuo C L et al 2008 Radiative emission and energy deposition in transient luminous events J. Phys. D Appl. Phys. 41234014

[52] Vallance Jones A 1974 Aurora ed D Reidel (Dordrecht: D. Reidel Publications) p $16+301$ 